\title{
Influence of methane addition on selenium sensitivity and spectral interferences
}

\author{
Geerke H. Floor ${ }^{\mathrm{a}, \mathrm{b}^{*}}$, Romain Millot ${ }^{\mathrm{a}}$, Mónica Iglesias ${ }^{\mathrm{b}}$, Philippe Négrel ${ }^{\mathrm{a}}$ \\ a) BRGM, Metrology Monitoring Analysis Department, Orléans, France \\ b) University of Girona, Department of Chemistry, Girona, Spain \\ * Corresponding author. Email: geerke.floor@aquatrain.eu
}

The measurements of stable Se isotopic signatures by multi-collector inductively coupled plasma mass spectrometry (MC-ICP-MS) are very challenging, due to the presence of spectral interferences and the low abundance of $\mathrm{Se}$ in environmental samples. We systematically investigated the effect of methane addition on the signal of selenium and its interferences. It is the first time that the effect of methane addition has been assessed for all Se isotopes and its potential interferences using hydride generator multi collector inductively coupled plasma mass spectrometry (HG-MC-ICP-MS). Our results show that a small methane addition increases the sensitivity. However, the response differs between a hydride generator and a standard introduction system, which might be related to differences in the ionization processes. Both argon and hydrogen based interferences, the most common spectral interferences on selenium isotopes in HG-MC-ICPMS, decrease with increasing methane addition. Therefore analyte-interference ratios and precision are improved. Methane addition has thus a high potential for the application to stable Se isotopes ratios by HG-MC-ICP-MS.

Keywords: selenium, isotopes, methane, multi collector ICPMS, interferences, precision, hydride generation 


\section{Introduction}

Selenium (Se) is an element with both environmental and toxicological interest, due to its narrow range between essential and poisonous concentrations. As for other elements, its speciation plays a key role in its toxicity and mobility ${ }^{1}$. Therefore, an understanding of the biogeochemical transformations of selenium is of primary importance. Selenium has six stable isotopes $(74,76,77,78,80$ and 82$)$ that may fractionate during biological and geochemical processes. ${ }^{1-12}$ Consequently, stable Se isotope measurements could potentially lead to a better understanding of Se mobility in the environment.

Selenium determinations are challenging for several reasons. Firstly, Se has a low abundance in geological matrixes. ${ }^{\text {e.g. } 1,9,13}$ For example the average concentration in upper continental crust is as low as $0.083 \mathrm{mg} \mathrm{kg}^{-1} .{ }^{14}$ Secondly, the sensitivity of Se is lower compared to most other elements in ICP techniques due to its high ionization energy that results in a poor ionization yield $(\approx 33 \%)$ in an argon plasma. ${ }^{13,15}$ Thirdly, four out of six Se isotopes $\left({ }^{74} \mathrm{Se},{ }^{76} \mathrm{Se},{ }^{77} \mathrm{Se},{ }^{82} \mathrm{Se}\right)$ have a low natural abundance $(<10 \%) .{ }^{16}$ Lastly, spectral interferences overlap with Se isotopes of which the plasma derived Ar polyatomic ions (on $\mathrm{m} / \mathrm{z}^{+} 76,78$ and 80 ) are a major concern. ${ }^{13,15,17,18}$

Isotopic measurements differ from concentration measurements because more interference free isotopes are needed. For element determination only the intensity of one single isotope (or two for quality control) is used. In contrast, for isotopic measurements a minimum of two (using standard bracketing for mass bias correction ${ }^{8,9,19}$ ) or four (using a double-spike for mass bias correction ${ }^{11,12,20}$ ) are needed. To plot the results on the mass fractionation line as control that interferences are well resolved, even one more interference isotope is required. Moreover, in MC-ICP-MS a total resolution of interferences is more important than in single collector ICP-MS, since a small deviation in signal intensity has a significant impact on the Se isotope ratio. Quadropole ICP-MS can be equipped with a reaction-collision cell to resolve interferences ${ }^{13,15,21-23}$. However, most multi-collector ICP-MS apparatuses lack the presence of a reaction-collision cell. Although high resolution has been used in Se determinations in sector field ICPMS, high resolution for Se isotopes in a multi-collector ICP-MS has never been reported to date, due to the small mass differences and the lower resolving power of multi collector compared to traditional high sector field ICP-MS ${ }^{13,24,25}$. Therefore, other approaches to improve the analyte-interference ratio are essential in isotopic measurements.

For selenium isotope ratio applications, high measurement precision is of extreme importance, because the variations in isotopic composition are very subtle (few promille). ${ }^{1-12}$ Precision is affected by the analyte signal (count statistics). Since Se levels are low in natural samples, improvement of Se sensitivity is therefore a major issue in isotopic Se measurements.

For above mentioned reasons, Se isotope measurements can be improved by increasing the Se sensitivity and increasing the analyte-interference ratio. An online hydride generator system coupled to the MC-ICPMS increases the sensitivity, because it significantly enhances the efficiency of the analyte transfer to the plasma and the ionization compared to standard wet aerosol introduction. ${ }^{8,9,11,12,26}$ Additional, the hydride generation only transfers volatile hydride forming elements to the plasma, thereby excluding some sample derived spectral interferences such as ${ }^{66} \mathrm{Zn}^{16} \mathrm{O}^{+},{ }^{48} \mathrm{Ti}^{14} \mathrm{~N}^{14} \mathrm{~N}$ and ${ }^{39} \mathrm{~K}^{39} \mathrm{~K} \cdot{ }^{13,20-27}$

On the other hand, carbon addition to the plasma shows beneficial effects on Se determination in ICP techniques. Both organic solvents added to the aqueous solutions or volatile carbon compounds introduced into the spray chamber can be used as carbon source. ${ }^{17,18,21,22,24,28-34}$ Carbon addition has two effects on Se determination. Firstly, a carbon loaded plasma increases the Se sensitivity. This 
enhancement is explained by several mechanisms, including a charge transfer reaction from $\mathrm{C}^{+}$-species to analyte atoms. ${ }^{17,28,31}$ Secondly, carbon loaded plasmas show lower levels of interferences, related to the competitive formation of carbides. ${ }^{17,18,29,30,32}$ Both effects increase the analyte-interference ratio. However, until now there is no systematic evaluation of the Se sensitivity and interferences for all Se isotopes.

In this study we asses the potential of a methane mixed plasma for the determination of Se isotopes using the MC-ICP-MS Neptune. For both a hydride generator and a standard introduction system, we studied the response of the Se signal on methane addition. To our knowledge no previous work exists on the combination of a methane-mixed plasma with a hydride generator introduction system. However, this is of great importance for the potential application to Se isotope measurements, since this is the common introduction system in Se isotopic analyses. Although the use of a hydride generator injector system eliminates some of the interferences, both argon based interferences (e.g. ArAr, $\mathrm{ArCl}, \mathrm{ArArH})$ and hydride forming elements (e.g. $\mathrm{Ge}, \mathrm{GeH}, \mathrm{SeH}$ and $\mathrm{AsH}$ ) still form interferences on Se isotopes. Therefore, we assessed the influence of methane addition on these interferences. Moreover, we compared the precision and mass bias between a methane mixed plasma and the traditional approach. As far as we are aware, it is the first time a carbon loaded plasma has been used in multi collector ICP-MS.

\section{Methods}

\subsection{Instrumentation and experimental conditions}

We performed all measurements with a NEPTUNE MC-ICP-MS (Finnigan, ThermoElectron). Two different introduction systems were used: 1) the ThermoFinnigan Stable Introduction System (SIS), which consists of a quartz glass dual spray chamber arrangement (cyclonic and standard Scott double pass) combined with a PFA® nebulizer ${ }^{35}$ and 2) a Hydride Generator Introduction System (HGIS). The sample gas was mixed with methane $\left(\mathrm{CH}_{4}=2.013 \pm 0.040 \mathrm{~mol} \%\right.$ in Ar) before the torch with a Yconnection part. For the SIS the sample was transferred to the plasma by self-aspiration (Figure 1a). For the hydride generator, the sample was pumped at a flow rate of about $0.25 \mathrm{ml} / \mathrm{min}$. The sample and reducing agent were merged at least $40 \mathrm{~cm}$ before they entered a gas-liquid separator to ensure good mixing. A low-flow of argon $(0.03 \mathrm{~L} / \mathrm{min})$ gas entered the gas-liquid separator in order to get efficient transport towards the plasma (indicated as Extra (Ar) in Figure 1b). The volatile selenium hydride was mixed with a sample gas flow, sometimes containing a small percentage of $\mathrm{CH}_{4}(<1 \%)$. Typical instrumental conditions for both set-ups are shown in Table 1. The ion optics and gas flows were daily tuned for optimum Se sensitivity without methane addition. After methane addition, only the sample gas flow and the radio frequency (RF) power were retuned for maximum sensitivity. For the comparison of final isotopic ratios we measured a $5 \mathrm{ppb}$ Se solution with and without methane for 10 minutes (using 150 integrations of 4 seconds each).

\subsection{Reagents and solutions}

For the SIS we used a Se solution (out of $1000 \mathrm{ppm} \mathrm{Se,} \mathrm{CPI} \mathrm{international)} \mathrm{with} \mathrm{a} \mathrm{concentration} \mathrm{one} \mathrm{order}$ of magnitude higher compared to the HGIS. However, the concentration used does not influence the results, as all data are normalized to the response without methane. Furthermore, the solution matrix was changed. For the SIS we used a $3 \%$ v/v nitric acid matrix (Merck Suprapure). $\mathrm{HCl}$ was avoided to 
maintain a low background level on $\mathrm{m} / \mathrm{z}^{+} 77$, otherwise influenced by ${ }^{40} \mathrm{Ar}^{37} \mathrm{Cl}^{+} \cdot{ }^{15,17}$ For the HGIS, we used $2 \mathrm{~N} \mathrm{HCl}$ (double distilled) in order to obtain efficient selenium-hydride formation. $\mathrm{HCl}$ has a strong reducing nature favouring the selenium hydride formation. ${ }^{17,26,36}$ With the use of a HGIS only part of the $\mathrm{Cl}$ will enter the plasma and cause a relatively small interference on $\mathrm{m} / \mathrm{z}^{+} 77 .{ }^{17}$ For the HGIS the reducing agent consisted of $0.5 \% \mathrm{w} / \mathrm{v} \mathrm{NaBH}_{4}$ (Merck reagent grade) stabilized by addition of $0.5 \% \mathrm{NaOH}$ (Merck reagent grade).

\section{Results and discussion}

\subsection{Se sensitivity: comparison between the introduction systems}

Changes in Se sensitivity due to the addition of methane are shown in Figure 2 for the SIS (circle) and the HGIS (square). The results are based on the measurements on $\mathrm{m} / \mathrm{z}^{+} 82$ in a Se solution, but other Se isotopes not (strongly) interfered (e.g. $\mathrm{m} / \mathrm{z}^{+} 77$ and 78) show a similar pattern. All results are normalized to the Se sensitivity without any methane addition. The maximum signal enhancement is around 2.5 at a methane flow rate of $4 \mathrm{~mL} \mathrm{~min}^{-1}$ for the SIS. For the HGIS the maximum signal enhancement is lower $(\sim 1.5)$ and at a lower flow rate $\left(\sim 1 \mathrm{~mL} \mathrm{~min}^{-1}\right)$. In this case methane addition above $2 \mathrm{~mL} \mathrm{~min}^{-1}$ gives lower sensitivity compared to no methane addition, while for the SIS the sensitivity increased for all used methane flows (up to $8 \mathrm{~mL} \mathrm{~min}^{-1}$ ). The sensitivity decrease for higher carbon loading with the use of organic solvents is thought to be related to the cooling of the plasma. ${ }^{31}$ However, this cannot explain the sensitivity decrease using volatile carbon compounds. Roduskhin et al [29] explained the signal decrease by higher diffusion losses of ions from the analytical zone in the methane-mixed plasma. Although slightly low, the enhancement factors fit within the range (between 1.5 and 9) previously reported. ${ }^{21,22,29,31}$ The different responses of the two injector systems might be related to the different forms of Se which enters to the plasma. For the SIS, the solution arrives as an aerosol. Consequently, some plasma energy is consumed for volatilization of the matrix and to convert the aerosols to gas. As a result, Se is not efficiently ionized. For the HGIS the Se enters the plasma as a gas. In this case the plasma energy is only needed for the atomization and ionization. Therefore, the ionization processes can be more efficient. The different response on the RF power for each introduction system confirms this hypothesis. The RF power is a parameter which is related to the temperature in the plasma. The optimum RF power without methane addition is higher for the SIS than for the HGIS (Table 1). Another difference is that for the SIS the RF optimum power depends on the amount of methane present in the plasma, while for the HGIS the optimum RF does not change with methane addition (Figure 3). The lower RF power for a carbon loaded plasma in the SIS, suggests that the methane facilitates the ionization ${ }^{26}$.

Another difference between the two systems concerns the sensitivity. Taking into account the different transport efficiencies and the flow rates, the Se loading towards the plasma vary about one order of magnitude between the two systems. However, the observed sensitivity difference is around 20-30 times. Thus, the discrepancy in sensitivity cannot be only explained by variations in the sample transfer and related Se loading within the plasma. This is an additional argument that differences in ionization efficiency occur between both systems. It also explains the dissimilarity in the enhancement factors between the two systems. Since the ionization of the SIS is less efficient, the ionization can be more improved than for the HG. Therefore, a higher enhancement factor is observed for SIS compared to HG. 


\subsection{Interferences on Se isotopes by HG-MC-ICP-MS}

Despite that the use of a hydride generator eliminates potential interferences on Se isotopes, several interferences can still occur (Table 2). In this section we discuss the effect of a methane mixed plasma on the occurrence of argon based interferences (e.g. Ar dimers, $\mathrm{ArCl}$ ), elements transferred to the plasma by the HGIS causing spectral interferences at Se isotopes (so called hydride forming elements, such as Ge, As) and other interferences (e.g. plasma derived $\mathrm{Kr}$, sample cone derived $\mathrm{NiO}$ ). It is essential to understand how methane influences these interferences, since even if interferences can be corrected, it is important to obtain the highest analyte-interference ratios possible. Although transition metals (e.g. $\mathrm{Fe}^{2+}$, $\mathrm{Cu}^{2+}$ and $\mathrm{Co}^{2+}$ ) can interfere with the hydride formation, these elements can easily be removed using a thiol-based sample preparation. ${ }^{9,25}$. Moreover, they do form matrix effects rather than spectral interferences on Se isotopes. Therefore, we have not further investigated the effects of these elements.

\subsubsection{Ar-based polyatomic ions}

Major $\mathrm{ArAr}^{+}$interferences occur at $\mathrm{m} / \mathrm{z}^{+} 76,78$ and $80{ }^{13,15,17,18}$ High resolution has never been performed for Se isotopes with MC-ICP-MS. Instruments equipped with a hexapole cell can eliminate the Ar interferences due to collisions with a reactive gas. ${ }^{8,9,19}$ However, most MC-ICP-MS do not contain a collision cell and therefore mathematical corrections for the Ar based interferences are required. The ${ }^{40} \mathrm{Ar}^{40} \mathrm{Ar}^{+}$has such a high intensity that precise ${ }^{80} \mathrm{Se}^{+}$data are excluded. On peak zero corrections (subtraction of signal intensities in blank solutions) almost completely removes the smaller $\mathrm{ArAr}^{+}$beams at $\mathrm{m} / \mathrm{z}^{+} 76$ and 78. However, a residual $\mathrm{ArAr}^{+}$intensity forms due to drift in the Ar dimers. ${ }^{11}$ These can be corrected using the measured intensity at $\mathrm{m} / \mathrm{z}^{+} 80$ and subtraction of the estimated Se intensity to obtain a calculated $\mathrm{ArAr}^{+}$intensity. This can be applied to calculate the Ar-dimer contribution at $\mathrm{m} / \mathrm{z}^{+} 76$ and 78. ${ }^{11,20}$ Although corrections exist, it is better to have the smallest Ar dimer beam as possible. Changes in Ar dimer signal due to the addition of methane are shown in Figure 4 (squares). The results are based on the measurements on $\mathrm{m} / \mathrm{z}^{+} 80$ in the blank solution $\left(2 \mathrm{~N} \mathrm{HCl}\right.$ ), whilst other $\mathrm{Ar}$ dimer masses (e.g. $\mathrm{m} / \mathrm{z}^{+} 76$ ) show a similar pattern. All results are normalized to the Ar dimer signal without any methane addition. The intensity of the Ar dimer decreases with increasing methane levels.

The $\mathrm{ArCl}^{+}$variation is minimized using matrix-matched blanks and solutions (within 5\%). Since the intensity of the $\mathrm{ArCl}^{+}$is small, an on peak zero correction is enough. ${ }^{11,25} \mathrm{We}$ monitored $\mathrm{m} / \mathrm{z}^{+} 77$ in a blank solution to investigate the effect of methane on the $\mathrm{ArCl}^{+}$. Although $\mathrm{ArArH}^{+}$also contributes to the 77 intensity, this beam is 2 orders of magnitude smaller compared to the $\mathrm{ArCl}^{+}$. Due to methane addition, the $\mathrm{ArCl}^{+}$decreases (circles in Figure 4).

The $\mathrm{Ar}_{2} \mathrm{H}^{+}$and $\mathrm{Ar}_{2} \mathrm{H}_{2}{ }^{+}$beams form interferences with respectively ${ }^{77} \mathrm{Se}^{+}$and ${ }^{82} \mathrm{Se}^{+}$. ArArH/ArAr ratios are normally below $1 \%$, depending on the tuning and daily conditions (data not shown). $\mathrm{Ar}_{2} \mathrm{H}_{2}{ }^{+}$is a small beam which can be corrected using on-peak zero corrections. Methane addition significantly decreases the intensity of $\mathrm{Ar}_{2} \mathrm{H}^{+} / \mathrm{ArAr}$ (monitored at $\mathrm{m} / \mathrm{z}^{+} 81 / 80$ corrected for ${ }^{81} \mathrm{Br}^{+}$using ${ }^{79} \mathrm{Br}^{+}$, rhombus in Figure 4) and therefore probably also for $\mathrm{Ar}_{2} \mathrm{H}_{2}{ }^{+}$.

The decrease of polyatomic interferences in carbon loaded plasmas have been observed before. ${ }^{17,18,20,29,30,34}$ This has been related to the competitive formation of carbides. The $\mathrm{ArArH}^{+}$beam decreases faster than for the $\mathrm{ArAr}^{+}$ion. The difference in signal decrease might be because three instead of two atoms are involved. Another reason can be that more signal decrease is observed for smaller signals. In both cases, the signal decrease on $\mathrm{Ar}_{2} \mathrm{H}_{2}{ }^{+}$is expected to be more than for $\mathrm{ArArH}^{+}$. 


\subsubsection{Hydride forming elements}

The hydride-forming ions such as arsenic (As) and germanium (Ge) form spectral interferences which hinder the measurements of Se isotopes. ${ }^{912,20}$ Therefore, it is important to know the effect of methane addition on the signal of $\mathrm{As}, \mathrm{Ge}, \mathrm{Br}$ and their hydride ions. Germanium can be quantitatively removed from the sample matrix using sample preparation. However, reagents can contain Ge. ${ }^{9,32}$ Mathematical corrections can be performed using the intensity on $\mathrm{m} / \mathrm{z}^{+} 72$ or 73 to calculate the $\mathrm{Ge}^{+}$contribution of $\mathrm{m} / \mathrm{z}^{+} 74$ and $\mathrm{m} / \mathrm{z}^{+} 76{ }^{11,20,23}$ Arsenic typically has a one or two order of magnitude higher concentration than Se in geological materials ${ }^{9}$. Although most As can be removed during the sample preparation step using thiol-cellulose/cotton, several percent of the initial As could remain into the solution. ${ }^{9,19,25}$ AsH corrections are difficult ${ }^{23}$, because AsH/As cannot be directly determined due to overlap with Ar dimers. However, in most samples after sample preparation As concentrations are so low that a correction is not required. ${ }^{11,23}$ However, for samples much more enriched in As than Se, such as shales and hydrothermal waters, interference corrections are needed. $\mathrm{SeH}^{+}$interferences at $\mathrm{m} / \mathrm{z}^{+} 77$ and 78 can be approximated using the intensity on $\mathrm{m} / \mathrm{z}^{+} 82$ and 83 to calculate the $\mathrm{SeH} / \mathrm{Se}$ ratio. ${ }^{11,12,20} \mathrm{HBr}^{+}$overlaps with Se isotopes at $\mathrm{m} / \mathrm{z}^{+} 80$ and 82 . However, only volatile species of bromide are transferred to the plasma using a hydride generator. ${ }^{11,20,23}$ Volatile $\mathrm{Br}$ species in samples can be removed by $\mathrm{N}_{2}$ bubbling ${ }^{12}$, eliminating the potential $\mathrm{HBr}^{+}$interference on Se isotopes. Although $\mathrm{m} / \mathrm{z}^{+} 79$ is monitored as a control, $\mathrm{HBr}^{+}$corrections are normally not needed. Therefore, we did not investigate the effect of methane on the HBr beam. Other hydride-forming elements such as $\mathrm{Te}, \mathrm{Sb}$ and $\mathrm{Sn}$ do not form spectral interferences with $\mathrm{Se}$ and are found not to have any adverse affect on the Se signal. ${ }^{9,16}$

The effect of methane addition on the hydride forming elements and their polyatomic ions are reported in Figure 5. It shows that the Ge signal is not enhanced, and therefore the relative influence of the Ge interference decreases. The optimum methane percentage for Se and As is the same. However, in agreement with previous work ${ }^{17,28,29,31}$, the As signal is more enhanced than Se. Although the As signal is more enhanced than the Se signal, we are only interested in AsH formation which interferes with the ${ }^{76} \mathrm{Se}$ isotope. As explained before, it is difficult to assess the AsH formation. However, we can estimate the $\mathrm{SeH}$ formation by monitoring $\mathrm{m} / \mathrm{z}^{+} 83$. The SeH intensity on $\mathrm{m} / \mathrm{z}^{+} 83$ decreases with methane addition (Figure 5). The SeH/Se ratio change depends on the relative change on Se intensity and $\mathrm{SeH}$ intensity. The hydride formation for Se decreased by a factor 0.7 at the optimum methane concentration whilst at this value As has around 1.2 times higher signal than Se. Therefore, when assuming a similar response for $\mathrm{AsH}$ as for $\mathrm{SeH}$, the estimated relative importance of $\mathrm{AsH}$ on $\mathrm{m} / \mathrm{z}^{+} 76$ is expected to decrease.

\subsubsection{Other interferences}

$\mathrm{Kr}$ interferences on Se isotopes occur at $\mathrm{m} / \mathrm{z}^{+} 78,80$ and 82. Trace $\mathrm{Kr}$ is present in the Ar gas used to generate the plasma. Moreover, $\mathrm{Kr}$ can dissolve from the atmosphere into sample solutions. ${ }^{11,19} \mathrm{Kr}$ interferences can be corrected measuring the intensity at $\mathrm{m} / \mathrm{z}^{+} 83$ or $84 .^{20,23}$ However, when samples and blanks are equilibrated with the atmosphere, an on peak-zero correction (subtraction of signal intensities in blank solutions) is normally sufficient. Nevertheless, it is useful to monitor the Kr intensity. ${ }^{11}$ The ${ }^{84} \mathrm{Kr}^{+}$beam decreases with methane addition (Figure 6). $\mathrm{Kr}$ has an ionization energy of $14.00 \mathrm{eV}$, which is above the ionization energy of $\mathrm{C}(11.20 \mathrm{eV})$. Previous work showed that no enhancement is observed for elements with an ionization energy above this threshold value because charge transfer reactions cannot occur. $^{28,31}$ 
The sample and skimmer cones are made of $\mathrm{Ni}$. NiO can overlap on different $\mathrm{m} / \mathrm{z}^{+} 74,76$ (and with low abundance also at $\mathrm{m} / \mathrm{z}^{+} 77,78$ and 80 ). However, these signals are very small and probably constant. It is difficult to assess the effect of methane on $\mathrm{NiO}$, because the beams are so small. Nevertheless, previous work shows that oxide levels decrease using a carbon loaded plasma. ${ }^{29,31,34}$

\subsection{Implications for Se isotope analyses}

Isotopic ratios corrected for the above discussed interferences are shown in Figure 7 for both with and without methane addition together with the theoretical mass fractionation line (exponential law). It shows that the same isotopic ratios $\left({ }^{76} \mathrm{Se} /{ }^{82} \mathrm{Se},{ }^{77} \mathrm{Se} /{ }^{82} \mathrm{Se}\right.$ and $\left.{ }^{78} \mathrm{Se} /{ }^{82} \mathrm{Se}\right)$ are obtained in both conditions. The fractionation from the natural isotopic abundance is a combination of natural fractionation (i.e. the isotopic signature of the sample) and the instrumental mass bias. Since in both cases we observe a similar fractionation and we used the same Se solution, we can conclude that methane addition does not significantly affect the instrumental mass bias. The precision of measurements was determined using signal monitoring during ten minutes (150 integrations of 4 seconds). The stability of the signal intensity is not better by the optimum methane addition $(1 \mathrm{~mL} / \mathrm{min})$. Although the count statistics are improved in a methane-mixed plasma due to higher sensitivity, the variations in the low gas flow of the methane-argon mixture induces an extra error. However, variations in signal intensity are compensated since all masses are collected simultaneously (static mode). Indeed, the isotopic ratios after interference correction show a better precision for a methane mixed plasma. Using the traditional approach a standard error of $\sim 0.2 \%$ o has been obtained. However, in the case of a methane mixed plasma the standard error is better $(\sim 0.07 \%$ ). This means that the standard error is decreased three times. The enhanced precision due to methane addition is related to the higher analyte-interference ratios.

\section{Conclusions}

We investigated the effect of methane addition on the sensitivity and interferences on all Se isotopes using a multi-collector ICP-MS. Firstly, the effect on Se sensitivity for both a standard and a hydride generator introduction system has been evaluated. The Se sensitivity is significantly enhanced for both introduction systems. However, for the standard introduction system (SIS), the maximum enhancement factor is higher and observed at a higher carbon loading compared to hydride generator introduction system (HGIS). This result can be explained by different ionization processes in both systems related to the Se form that enters into the plasma. This is also confirmed by differences in the optimum RF power (associated with the plasma temperature) and different sensitivities that are not related to changes in Se loading.

Secondly, the effect of methane addition on common interferences in HG-MC-ICP-MS has been assessed. Intensities of polyatomic interferences (such as $\mathrm{ArAr}, \mathrm{ArCl}$ and several hydride containing species) decrease with methane addition, due to the competitive formation of carbides. Germanium also decreases its intensity. Arsenic intensity is more enhanced than Se, however, the AsH beam is probably less enhanced than the Se signal.

Thirdly, interference corrected Se isotopic ratios, mass bias and precision have been compared in both measurement conditions. Corrected isotopic ratios and mass bias are similar with and without methane addition. However, the precision is improved three times due to a better signal-blank ratio. 
Therefore, we can conclude from this study that methane addition has a high potential for the determination of $\mathrm{Se}$ isotope ratios. The Se sensitivity is increased, while the intensities of the interferences are considerably decreased. These improvements are very important for the measurement of stable Se isotopes in environmental samples, which can significantly enhance our knowledge about Se cycling in the environment.

\section{Acknowledgements}

We would like to acknowledge M. Robert for her help in the Neptune laboratory. Thanks to T.M. Johnson for providing the hydride generator. T.D. Bullen and T.M. Johnson are acknowledged for discussions. Thanks to M. Kuijpers for her help with Figure 1 and to A. Hegan for critical reading of the manuscript. This is a contribution of the AquaTRAIN Marie Curie Research Training Network (Contract No. MRTN-CT-2006-035420) funded under the European Commission Sixth Framework Programme (20022006) Marie Curie Actions, Human Resources \& Mobility Activity Area - Research Training Networks. It reflects the views of the authors but not necessarily those of the European Community, which is not liable for any use that may be made of the information contained therein. Additional funding was obtained from the Research Division of BRGM. This is BRGM contribution $n^{\circ} 6437$.

\section{References}

1. T. M. Johnson, M. J. Herbel, T. D. Bullen and P. T. Zawislanski, Fractionation of selenium isotopes during bacterial respiratory reduction of selenium oxyanions, Geochim Cosmochim Ac, 63 (1999), 2775-2783.

2. T. M. Johnson, T. D. Bullen and P. T. Zawislanski, Selenium stable isotope ratios as indicators of sources and cycling of selenium: Results from the northern reach of San Francisco Bay, Environ Sci Technol, 34 (2000), 2075-2079.

3. T. M. Johnson, A review of mass-dependent fractionation of selenium isotopes and implications for other heavy stable isotopes Chem Geol, 204 (2004), 201-214.

4. T. M. Johnson and T. D. Bullen, Selenium isotope fractionation during reduction by Fe(II)-Fe(III) hydroxide-sulfate (green rust). Geochim Cosmochim Ac, 67 (2003), 413-419.

5. T. M. Johnson and T. D. Bullen, Mass-Dependent Fractionation of Selenium and Chromium Isotopes in LowTemperature Environments, Rev Min Geochem, 55 (2004), 289-317.

6. M. J. Herbel, T. M. Johnson, R. S. Oremland and T. D. Bullen, Fractionation of selenium isotopes during bacterial respiratory reduction of selenium oxyanions, Geochim Cosmochim Ac, 64 (2000), 3701-3709.

7. M. J. Herbel, T. M. Johnson, K. K. Tanji, S. D. Gao and T. D. Bullen, Selenium stable isotope ratios in California agricultural drainage water management systems, Journal of Environ Qual, 31 (2002), 1146-1156.

8. O. Rouxel, Y. Fouquet and J. N. Ludden, Subsurface processes at the lucky strike hydrothermal field, Mid-Atlantic ridge: evidence from sulfur, selenium, and iron isotopes, Geochim Cosmochim Ac, 68 (2004), 2295-2311.

9. O. Rouxel, J. Ludden, J. Carignan, L. Marin and Y. Fouquet, Natural variations of Se isotopic composition determined by hydride generation multiple collector inductively coupled plasma mass spectrometry, Geochim Cosmochim Ac, 66 (2002), 3191-3199.

10. A. S. Ellis, T. M. Johnson, M. J. Herbel and T. D. Bullen, Stable isotope fractionation of selenium by natural microbial consortia, Chem Geol, 195 (2003), 119-129.

11. S. K. Clark and T. M. Johnson, Effective Isotopic Fractionation Factors for Solute Removal by Reactive Sediments: A Laboratory Microcosm and Slurry Study, Environ Sci Technol, 42 (2008), 7850-7855.

12. J.-M. Zhu, T. M. Johnson, S. K. Clark and X.-K. Zhu, High Precision Measurement of Selenium Isotopic Composition by Hydride Generation Multiple Collector Inductively Coupled Plasma Mass Spectrometry with a 74Se-77Se Double Spike, Chinese J Anal Chem, 36 (2008), 1385-1390.

13. N. Elwaer and H. Hintelmann, Comparing the precision of selenium isotope ratio measurements using collision cell and sector field inductively coupled plasma mass spectrometry, Talanta, 75 (2008), 205-214

14. K. Hans Wedepohl, The composition of the continental crust., Geochim Cosmochim Ac, 59 (1995), 1217-1232.

15. W. O. Moellmer and T. G. Miller, ICP-MS Analysis of Trace Selenium in the Great Salt Lake, Spectroscopy (2007) January, 6.

16. N. Elwaer and H. Hintelmann, Comparative performance study of different sample introduction techniques for rapid and precise selenium isotope ratio determination using multi-collector inductively coupled plasma mass spectrometry (MCICP/MS), Anal BioAnal Chem, 389 (2007), 1889-1899.

17. R. Muñoz-Olivas, C. R. Quétel and O. F. X. Donard, Sensitive determination of selenium by inductively coupled plasma mass spectrometry with flow injection and hydride generation in the presence of organic solvents, J. Anal. At. Spectrom, 10 (1995), $865-870$.

18. E. H. Evans and L. Ebdon, Interferences in inductively coupled plasma mass spectrometry. A review, J. Anal. At. Spectrom, 5 (1990), 425-430.

19. J. Carignan and H. Wen, Scaling NIST SRM 3149 for Se isotope analysis and isotopic variations of natural samples, Chem Geol, 242 (2007), 347-350.

20. N. Elwear and H. Hintelmann, Selective separation of selenium (IV) by thiol cellulose powder and subsequent selenium isotope ratio determination using multicollector inductively coupled plasma mass spectrometry, J. Anal. At. Spectrom, 23 (2008), 733-743

21. E. Warburton and H. Goenage-Infante, Methane mixed plasma-improved sensitivity of inductively coupled plasma mass spectrometry detection for selenium speciation analysis of wheat-based food, J. Anal. At. Spectrom, 22 (2007), 370-376

22. H. Goenaga Infante, M. Ovejero Bendito, C. Cámara, L. Evans, R. Hearn and S. Moesgaard, Isotope dilution quantification of ultratrace gamma-glutamyl-Se-methylselenocysteine species using HPLC with enhanced ICP-MS detection by ultrasonic nebulisation or carbon-loaded plasma , Anal BioAnal Chem, 390 (2008), 2099-2106. 
23. D. Layton-Matthews, M. I. Leybourne, J. M. Peter and S. D. Scotta, Determination of selenium isotopic ratios by continuous-hydride-generation dynamic-reaction-cell inductively coupled plasma-mass spectrometry, J. Anal. At. Spectrom, 21 (2005), 41-49.

24. E. H. Larsen and S. Sturup, Carbon-enhanced Inductively Coupled Plasma Mass Spectrometric Detection of Arsenic and Selenium and Its Application to Arsenic Speciation, J. Anal. At. Spectrom, 9 (1994), 1099-1105.

25. F. Vanhaecke, L. Balcaen and D. Malinovsky, Use of single-collector and multi-collector ICP-mass spectrometry for isotopic analysis. J. Anal. At. Spectrom 24(2009), 863-886.

26. J. D. Hwang, G.D Guenther and J.P. Diomiguardi A hydride generator system for a 1-kW inductively coupled plasma, Anal Chem, 61 (1989), 285-288

27. G.H. Floor, M. Iglesias, G. Román-Ross. Selenium determination in volcanic soils by ICP-QMS: influence of reaction cell pressurization and methanol addition on the occurrence of spectral interferences. J Anal At Spectrom 24 (2009), 944948 .

28. P. Allain, L. Jaunault, Y. Mauras, J. M. Mermet and T. Delaporte, Signal enhancement of elements due to the presence of carbon-containing compounds in inductively coupled plasma mass spectrometry , Anal Chem, 63 (1991), 1497-1498.

29. I. Rodushkin, P. Nordlund, E. Engstrøm and D. C. Baxter, Improved multi-elemental analyses by inductively coupled plasma-sector field mass spectrometry through methane addition to the plasma , J. Anal. At. Spectrom, 20 (2005), 12501255 .

30. S. J. Hill, M. J. Ford and L. Ebdon, Investigations into the application of methane addition to the nebulizer gas in inductively coupled plasma mass spectrometry for the removal of polyatomic interferences, J. Anal. At. Spectrom, 7 (1992), 1157-1165.

31. Z. Hu, S. Hu, S. Gao, Y. Liu and S. Lin, Volatile organic solvent-induced signal enhancements in inductively coupled plasma-mass spectrometry: a case study of methanol and acetone, Spectrochim. Acta, B, 59 (2004), 1463-1470.

32. E. H. Evans and J. J. Giglio, Effect of Organic Solvents and Molecular Gases on Polyatomic Ion Interferences in Inductively Coupled Plasma Mass Spectrometry., J. Anal. At. Spectrom, 8 (1993), 1-18.

33. K.S. Park, S.-T. Kim, Y.-M. Kim, Y. Kim and W. Lee, Application of methane mixed plasma for the determination of Ge, As, and Se in serum and urine by ICP/MS, B Kor Chem Soc 24 (2003), 285-290.

34. L. Ebdon, M. J. Ford, R. C. Hutton and S. J. Hill, Evaluation of Ethene Addition to the Nebulizer Gas in Inductively Coupled Plasma-Mass Spectrometry for the Removal of Matrix-, Solvent-, and Support-Gas-Derived Polyatomic Ion Interferences, Appl Spectrosc, 48 (1994), 507-516.

35. S. Weyer and J. B. Schwieters, High precision Fe isotope measurements with high mass resolution MC-ICPMS." International Journal of Mass Spectrometry , Int J Mass Spectrom, 226 (2003), 355-368.

36. M. Verlinden, J. Baart and H. Deelstra, Optimization of the determination of selenium by atomic-absorption spectrometry: Comparison of two hydride generation systems , Talanta, 27 (1980), 633-639 


\section{Table captions}

Table 1: Instrumental conditions

Table 2: Se isotopes and their potential interferences using a hydride generator injection system

\section{Figure captions}

Figure 1: experimental set-up a) for a stable introduction system and b) for a hydride generator introduction system. $S=$ sample; $R=$ reducing agent; $W=$ waste; $F=$ filter.

Figure 2: The effect of methane addition on Se sensitivity

Figure 3: The effect of RF power for Stable Introduction System (SIS) and hydride generator Introduction System (HGIS) for different carbon loading. The intensity is normalized to optimum settings without methane addition

Figure 4: The response of Ar-based polyatomic interferences on methane addition. Grey bar indicated optimum methane flow (with methane in Figure 7).

Figure 5: The responses of hydride forming elements on methane addition. Grey bar indicated optimum methane flow (with methane in Figure 7).

Figure 6: The response of $\mathrm{Kr}$-intensity on methane addition. Grey bar indicated optimum methane flow (with methane in Figure 7).

Figure 7: Interference corrected Se isotopic ratios ${ }^{76} \mathrm{Se} /{ }^{82} \mathrm{Se}$ and ${ }^{77} \mathrm{Se} /{ }^{82} \mathrm{Se}$ vs. ${ }^{78} \mathrm{Se} /{ }^{82} \mathrm{Se}$ with and without methane addition. Open symbols: ${ }^{76} \mathrm{Se} /{ }^{82} \mathrm{Se}$. Closed symbols: ${ }^{77} \mathrm{Se} /{ }^{82} \mathrm{Se}$. 